\title{
Prenatal Nicotine Exposure Alters the Response to Nicotine Administration in Adolescence: Effects on Cholinergic Systems During Exposure and Withdrawal
}

\author{
Yael Abreu-Villaça',2, Frederic J Seidler', Charlotte A Tate', Mandy M Cousins' and Theodore A Slotkin*,' \\ 'Department of Pharmacology and Cancer Biology, Duke University Medical Center, Durham, NC 277I 0, USA; ${ }^{2}$ Faculdade de Ciências Médicas, \\ Fundação Educacional Serra dos Órgãos (FESO), Teresópolis, Rio de Janeiro, Brazil
}

\begin{abstract}
Maternal smoking during pregnancy increases the likelihood that the offspring will become smokers in adolescence. In the current study, we evaluated effects of prenatal and adolescent nicotine exposure in rats to assess whether there is a biological basis for this relationship. Pregnant rats were given nicotine or vehicle throughout pregnancy and the offspring then again received nicotine or vehicle during adolescence (postnatal days PN30-47.5), using a regimen $(6 \mathrm{mg} / \mathrm{kg} /$ day by subcutaneous infusion) that produces plasma nicotine levels similar to those in smokers. Evaluations were made in the cerebral cortex and midbrain during adolescent nicotine administration (PN45) and for up to I month after the end of treatment. We assessed the magnitude and persistence of nicotinic acetylcholine receptor (nAChR) upregulation; in addition, we evaluated cholinergic synaptic activity by comparing the effects on choline acetyltransferase (ChAT), a constitutive marker for cholinergic nerve terminals, with those on hemicholinium-3 (HC-3) binding to the presynaptic choline transporter, which is regulated by nerve impulse activity. Prenatal nicotine exposure had only minor effects on nAChRs but produced persistent cholinergic hypoactivity (reduced HC-3 binding relative to ChAT) throughout adolescence and into adulthood (PN75). Adolescent nicotine exposure evoked robust nAChR upregulation and also suppressed cholinergic activity. Prenatal nicotine exposure reduced the upregulation of $n A C h R s$ evoked by adolescent nicotine but worsened the cholinergic hypoactivity during withdrawal. Our results indicate that prenatal nicotine exposure alters the subsequent response to nicotine in adolescence, effects that may contribute to the association between maternal smoking during pregnancy and subsequent adolescent smoking in the offspring.

Neuropsychopharmacology (2004) 29, 879-890, advance online publication, I 8 February 2004; doi: I0.1038/sj.npp. I30040 I
\end{abstract}

Keywords: acetylcholine; adolescence; brain development; choline acetyltransferase; choline transporter; cholinergic; hemicholinium-3 binding; nicotine; nicotinic cholinergic receptors

\section{INTRODUCTION}

Most smokers begin the habit in teen or preteen years (Nelson et al, 1995; Pierce and Gilpin, 1996), and initiation of smoking during this period is associated with greater daily consumption, a higher probability of addiction, and a lower probability of quitting (Chassin et al, 1996; Chen and Millar, 1998). Recent work with animal models of nicotine administration suggests that the greater susceptibility of adolescents has a biological basis. Exposure of adolescent rats to plasma levels of nicotine found in regular smokers elicits greater and more persistent nicotinic acetylcholine receptor (nAChR) upregulation than that found in adults, and the adolescent effects are accompanied by prolonged

*Correspondence: Dr TA Slotkin, Box 38 I3 DUMC, Duke Univ. Med. Ctr., Durham, NC 277I0, USA, Tel: + I 919681 8015, Fax: + I919 684 8197, E-mail: t.slotkin@duke.edu

Received 04 November 2003; revised 15 December 2003; accepted 05 January 2004

Online Publication: 7 January 2004 at http://www.acnp.org/citations/ Npp010704035 I/default.pdf suppression of cholinergic function as well as outright brain cell damage, typified by neuronal loss, apoptosis, and alterations in synaptic connectivity (Abreu-Villaça et al, 2003a, c; Slotkin, 2002; Trauth et al, 1999, 2000a, 2001). In fact, even lower adolescent nicotine exposures, down to one-tenth those found in smokers, are capable of eliciting these changes (Abreu-Villaça et al, 2003a, c). At the behavioral level, the effects of adolescent nicotine are also distinct from those seen in adults (Adriani et al, 2002, 2003; Faraday et al, 2001, 2003; Levin, 1999; Trauth et al, 2000c) including persistent changes in EEG consistent with neural cell damage (Slawecki and Ehlers, 2002).

There are subsets of adolescent smokers that exhibit different degrees of susceptibility to nicotine addiction. Approximately one-fourth of teenage smokers will not become daily smokers (Centers for Disease Control and Prevention, 1998; National Institute on Drug Abuse, 1998; Nelson et al, 1995) and, whereas some individuals show very rapid onset of dependence, others require more prolonged exposure and higher consumption (DiFranza et al, 2000, 2002a,b). Among the factors that may 
predispose adolescent smokers to nicotine addiction, parental smoking provides an important contribution (Bauman et al, 1990; Chassin et al, 2002; Niaura et al, 2001). Perhaps most importantly, maternal smoking during pregnancy is a strong predictor of subsequent smoking in adolescent offspring, regardless of whether parental smoking continues during childhood (Cornelius et al, 2000; Kandel et al, 1994; Niaura et al, 2001). Again, these point to a biological basis for prenatally induced differences in subsequent susceptibility to adolescent nicotine addiction. It is well-established that nicotine is a neuroteratogen that elicits neural cell death, alters cell proliferation and differentiation, and evokes deficits in lasting deficits in synaptic function and behavioral performance (reviews, Levin and Slotkin, 1998; Slotkin, 1992, 1998, 1999, 2004). However, there is scant information as to whether prenatal nicotine exposure alters the subsequent biological response to nicotine. In two earlier studies, we found lasting cholinergic hypoactivity (Zahalka et al, 1992) and desensitization of catecholamine release in response to acute nicotine challenge (Seidler et al, 1992); these observations were reinforced by a recent report from another laboratory, showing that prenatal nicotine exposure influences nicotine self-administration in adolescent mice (Klein et al, 2003).

Accordingly, the current study assesses the effects of prenatal nicotine exposure of rats on the response to nicotine administered in adolescence, using continuous infusions via implanted osmotic minipumps to achieve nicotine plasma levels found in typical smokers (Lichtensteiger et al, 1988; Murrin et al, 1987; Ostrea et al, 1994; Trauth et al, 2000b). In keeping with earlier experimental designs (Abreu-Villaça et al, 2003a-c; Levin and Slotkin, 1998; Slotkin, 1992, 1998, 1999, 2004), prenatal exposure was conducted throughout gestation, with subsequent adolescent exposure for just over 2 weeks beginning on postnatal day (PN) 30. Evaluations were conducted during adolescent exposure and for up to 1 month after the initiation of withdrawal. We evaluated three cholinergic biomarkers that delineate the greater sensitivity of the adolescent brain to nicotine (Abreu-Villaça et al, 2003a; Navarro et al, 1989; Trauth et al, 2000a; Zahalka et al, 1992). First, we assessed nAChR binding with $\left[{ }^{3} \mathrm{H}\right]$ cytisine, a ligand that binds selectively to the $\alpha 4 \beta 2 \mathrm{nAChR}$, the predominant subtype in mammalian brain that is robustly upregulated by nicotine administration (Flores et al, 1992; Happe et al, 1994; Trauth et al, 1999; Whiting and Lindstrom, 1987, 1988). We also assessed choline acetyltransferase activity (ChAT) and the binding of $\left[{ }^{3} \mathrm{H}\right]$ hemicholinium-3 (HC-3) to the high-affinity presynaptic choline transporter. ChAT, the enzyme responsible for acetylcholine biosynthesis, is a constitutive marker for cholinergic nerve terminals and serves as an archetypal measure of cholinergic innervation, but its activity does not respond to changes in impulse flow. Accordingly, ChAT reflects the concentration of cholinergic nerve terminals but does not change in response to stimuli that alter cholinergic neuronal activity (Aubert et al, 1996; Happe and Murrin, 1992; Navarro et al, 1989; Slotkin et al, 1990; Zahalka et al, 1992, 1993). In contrast, high affinity choline uptake, as assessed with the binding of $\mathrm{HC}-3$ to the presynaptic high-affinity choline transporter, is responsive to neuronal activity (Klemm and Kuhar, 1979; Simon et al, 1976) and the comparative changes in ChAT and HC-3 binding or transporter function (HC-3/ChAT ratio) permit distinction between effects on the concentration of synaptic terminals as distinct from those on synaptic activity (Aubert et al, 1996; Bissette et al, 1996; Happe and Murrin, 1992; Navarro et al, 1989; Slotkin et al, 1990; Zahalka et al, 1992, 1993).

\section{MATERIALS AND METHODS}

\section{Animals and Nicotine Infusions}

All studies were carried out with the approval of the Duke University Institutional Animal Care and Use Committee, in accordance with the declaration of Helsinki and with the Guide for the Care and Use of Laboratory Animals as adopted and promulgated by the National Institutes of Health. Timed-pregnant Sprague-Dawley rats were shipped on gestational day (GD) 2 by climate-controlled truck (total transit time less than $1 \mathrm{~h}$ ), housed individually, and allowed free access to food and water. There were four treatment groups: controls (prenatal vehicle + adolescent vehicle), prenatal nicotine exposure (prenatal nicotine + adolescent vehicle), adolescent nicotine exposure (prenatal vehicle + adolescent nicotine), and those receiving the combined treatment (nicotine both during prenatal development and adolescence). On GD4, before implantation of the embryo in the uterine wall, each dam was lightly anesthetized with ether, a $3 \times 6 \mathrm{~cm}$ area on the back was shaved, and an incision made to permit s.c. insertion of type 2ML2 Alzet osmotic minipumps. Pumps were prepared with nicotine bitartrate dissolved in bacteriostatic water, to deliver an initial dose rate of $6 \mathrm{mg} / \mathrm{kg}$ of nicotine (calculated as free base) per day. The incision was closed with wound clips and the animals were permitted to recover in their home cages. Control animals were implanted with minipumps containing only the water and an equivalent concentration of sodium bitartrate. It should be noted that the pump, marketed as a 2-week infusion device, actually takes 17.5 days to be exhausted completely (information supplied by the manufacturer) and thus the nicotine infusion terminates during GD21. The characteristics of this administration model have been reviewed previously (Levin and Slotkin, 1998; Slotkin, 1992, 1998, 1999, 2004).

Parturition occurred during GD22, which was also taken as PN0. After birth, pups were briefly removed from the dams, all animals within a given treatment group were placed together, and then the pups were reassigned to dams randomly. Litter sizes were culled to 10 (five males and five females) to ensure standard nutrition. Randomization was repeated every 3-4 days to distribute differential effects of maternal caretaking equally among all litters. Crossfostering, by itself, has no impact on neurochemical or behavioral effects of nicotine exposure (Ribary and Lichtensteiger, 1989). Animals were weaned on PN21.

On PN30, each animal was implanted with a minipump (Alzet type, 1002) as already described, again set to deliver either vehicle or nicotine at an initial dose rate of $6 \mathrm{mg} / \mathrm{kg}$ per day, with the infusion terminating during PN47 (Slotkin, 1998; Trauth et al, 1999, 2000b). In the adolescent rat this paradigm produces plasma nicotine levels of $25 \mathrm{ng} /$ $\mathrm{ml}$, similar to that in typical smokers (Lichtensteiger et al, 1988; Trauth et al, 2000b); it produces a transient (10\%) 
reduction in body weight during treatment, an effect that disappears after termination of the infusion (Abreu-Villaça et al, 2003a; Trauth et al, 1999). Studies were conducted at four time points, one during adolescent nicotine administration (PN45) and three during the withdrawal period (PN50, PN60 and PN75). Animals were decapitated and the cerebral cortex and midbrain were dissected (Trauth et al, $2000 \mathrm{~b}$ ), frozen in liquid nitrogen, and stored at $-45^{\circ} \mathrm{C}$ until assayed. For each treatment group, 12 animals were examined at each age point, equally divided into males and females.

\section{Tissue Preparation and Assays}

Tissues were thawed and homogenized (Polytron, Brinkmann Instruments, Westbury, NY) in approximately 40 volumes of ice-cold $50 \mathrm{mM}$ Tris $\mathrm{HCl}(\mathrm{pH} \mathrm{7.4)}$ ), and aliquots were withdrawn for measurements of ChAT activity (Lau et al, 1988) and total protein (Smith et al, 1985). To prepare the cell membrane fraction, the homogenates were sedimented at $40000 \mathrm{~g}$ for $10 \mathrm{~min}$ and the supernatant solution was discarded. The membrane pellet was resuspended (Polytron) in the original volume of buffer, resedimented, and the resultant pellet was resuspended in approximately 10 volumes (based on the original weight of the tissue) of the same buffer using a smooth glass homogenizer fitted with a Teflon pestle. Aliquots of this resuspension were withdrawn for measurements of $\left[{ }^{3} \mathrm{H}\right]$ cytisine and $\left[{ }^{3} \mathrm{H}\right] \mathrm{HC}-3$ binding, and for membrane protein.

All assays have been described in detail in our previous papers (Abreu-Villaça et al, 2003a; Trauth et al, 1999, 2000a) and will therefore be presented briefly. ChAT activity was assayed in tissue homogenate using $50 \mu \mathrm{M}\left[{ }^{14} \mathrm{C}\right]$ acetylcoenzyme A and labeled acetylcholine was then extracted and the activity determined relative to tissue protein. $\left[{ }^{3} \mathrm{H}\right]$ Cytisine and $\left[{ }^{3} \mathrm{H}\right] \mathrm{HC}-3$ binding were determined in the cell membrane fraction using final ligand concentrations of 1 and $2 \mathrm{nM}$, respectively; specific binding was displaced with $20 \mu \mathrm{M}$ nicotine for nAChRs and $10 \mu \mathrm{M}$ unlabeled HC-3 for the high affinity choline transporter. Values were then calculated as specific binding per mg of membrane protein.

\section{Data Analysis}

To reduce the likelihood of type 1 statistical errors that might result from repeated testing of the global data set, we first performed a global analysis of variance (ANOVA) (with log-transformed data because of heterogeneous variance) incorporating all the variables and measures: prenatal treatment, adolescent treatment, brain region, age, sex, and the three cholinergic biomarkers (nAChR binding, ChAT, HC-3 binding). The biomarkers were treated as repeated measures, since each was obtained from the same tissue homogenate. This initial test indicated treatment effects that differed among the different biomarkers, so data were then examined separately for each measure, again using a multivariate ANOVA (prenatal treatment, adolescent treatment, region, age, sex). Where appropriate, this was followed by post hoc evaluations of each treatment group compared with the controls, using Fisher's Protected Least Significant Difference; however, where treatment effects did not interact with other variables only the main effect was recorded without testing of individual differences. Effects of nicotine on body weights were conducted similarly with a multivariate ANOVA (prenatal treatment, adolescent treatment, age, sex) using log-transformed values; brain region weights were evaluated with the additional factor of region. Significance was assumed at the level of $p<0.05$ for main effects; however, for interactions at $p<0.1$, we also examined whether lowerorder main effects were detectable after subdivision of the interactive variables (Snedecor and Cochran, 1967).

The study design required two different ways of regarding treatment variables. To compare the effects of prenatal exposure alone, adolescent exposure, or the combined exposure to controls or to each other, the four treatment groups were considered as a one-dimensional factor in the statistical design. To determine whether the effects of prenatal exposure and adolescent exposure were interactive, the treatment factors were changed to a two-dimensional design. In this formulation, more-than additive (synergistic) and less-than additive effects appear as significant interactions between the two treatment dimensions, whereas simple, additive effects do not show significant interactions.

Data are presented as means and standard errors. To facilitate comparisons across multiple tissues, ages, and variables, the effects of each treatment are given as the percentage change from the corresponding control group, but statistical comparisons were made on the original data. For reference, control values appear in Table 1.

\section{Materials}

Animals were purchased from Charles River (Raleigh, NC) and osmotic minipumps from Durect Corp. (Cupertino, CA). Bacteriostatic water was obtained from Abbott Laboratories (N. Chicago, IL). Radioisotopically-labeled compounds came from Perkin-Elmer Life Sciences (Boston, MA): $\left[{ }^{14} \mathrm{C}\right]$ acetyl-CoA (specific activity $44 \mathrm{mCi} / \mathrm{mmol}$, diluted with unlabeled compound to $6.7 \mathrm{mCi} / \mathrm{mmol}),\left[{ }^{3} \mathrm{H}\right] \mathrm{HC}-3$ (specific activity $128 \mathrm{Ci} / \mathrm{mmol}$ ) and $\left[{ }^{3} \mathrm{H}\right]$ cytisine (specific activity, $35 \mathrm{Ci} / \mathrm{mmol}$ ). Sigma Chemical Co. (St Louis, MO) was the source for all other reagents.

\section{RESULTS}

Across all treatment groups and ages, ANOVA indicated main effects of nicotine treatment on body weight as well as interactions of treatment with age and sex (Figure 1). As the main effects was significant and robust for both males $(p<0.0001)$ and females $(p<0.002)$, differing only in the time course of effect $(p<0.03$ for treatment $\times$ age in males; no treatment $\times$ age interaction in females), values were combined across sex to simplify the presentation. Prenatal nicotine exposure elicited a small (5\%), but statistically significant elevation in body weight on PN30 and 37, with values gradually returning to normal over the next few weeks. In keeping with our earlier results for the adolescent nicotine model (Trauth et al, 1999, 2000b), body weights were reduced slightly during the 2-week period of treatment and returned to normal shortly thereafter. Prenatal nicotine exposure did not alter the subsequent weight loss caused by adolescent nicotine treatment. The group receiving the 
Table I Control Values for Cholinergic Biomarkers

\begin{tabular}{|c|c|c|c|c|c|c|c|c|c|c|c|c|}
\hline \multirow{5}{*}{ Body weight (g) } & \multicolumn{2}{|c|}{ PN30 } & \multicolumn{2}{|c|}{ PN37 } & \multicolumn{2}{|c|}{ PN45 } & \multicolumn{2}{|c|}{ PN50 } & \multicolumn{2}{|c|}{ PN60 } & \multicolumn{2}{|c|}{ PN75 } \\
\hline & Male & Female & Male & Female & Male & Female & Male & Female & Male & Female & Male & Female \\
\hline & $95 \pm 1$ & $87 \pm 1 *$ & $165 \pm 2$ & $135 \pm 2 *$ & $240 \pm 3$ & $176 \pm 3 *$ & $286 \pm 5$ & $194 \pm 4 *$ & $360 \pm 8$ & $224 \pm 4 *$ & $453 \pm 18$ & $259 \pm 7 *$ \\
\hline & \multirow[b]{2}{*}{ Region } & \multicolumn{2}{|c|}{ PN45 } & \multicolumn{2}{|c|}{ PN50 } & \multicolumn{2}{|c|}{ PN60 } & \multicolumn{2}{|c|}{ PN75 } & & & \\
\hline & & Male & Female & Male & Female & Male & Female & Male & Female & & & \\
\hline \multirow[t]{2}{*}[{}^{3}\mathrm{H}]{ Cytisine binding (fmol/mg protein) } & Cerebral cortex & $69 \pm 3$ & $66 \pm 3$ & $68 \pm 4$ & $68 \pm 3$ & $69 \pm 4$ & $71 \pm 4$ & $61 \pm 2$ & $65 \pm 3$ & & & \\
\hline & Midbrain & $91 \pm 4$ & $106 \pm 5^{*}$ & $85 \pm 5$ & $89 \pm 5$ & $76 \pm 3$ & $79 \pm 2$ & $86 \pm 6$ & $99 \pm 4$ & & & \\
\hline \multirow[t]{2}{*}{ ChAT activity (pmol/min/mg protein) } & Cerebral cortex & $615 \pm 21$ & $659 \pm 28$ & $740 \pm 20$ & $756 \pm 38$ & $710 \pm 36$ & $760 \pm 28$ & $722 \pm 12$ & $78 \mid \pm 18^{*}$ & & & \\
\hline & Midbrain & $579 \pm 19$ & $623 \pm 24$ & $724 \pm 25$ & $761 \pm 15$ & $652 \pm 25$ & $7 \mid 4 \pm 24$ & $627 \pm 20$ & $650 \pm 5$ & & & \\
\hline \multirow[t]{2}{*}[{}^{3}\mathrm{H}]{$\mathrm{HC}-3$ binding (fmol/mg protein) } & Cerebral cortex & $11.7 \pm 0.5$ & $12.1 \pm 0.7$ & $10.8 \pm 0.9$ & $10.8 \pm 1.2$ & $12.6 \pm 0.8$ & $11.1 \pm 0.7$ & $11.0 \pm 0.6$ & $10.6 \pm 0.5$ & & & \\
\hline & Midbrain & $8.8 \pm 0.7$ & $9.2 \pm 0.4$ & $9.5 \pm 0.5$ & $8.8 \pm 0.5$ & $8.1 \pm 0.4$ & $7.5 \pm 0.3$ & $7.7 \pm 0.5$ & $7.0 \pm 0.5$ & & & \\
\hline \multirow[t]{2}{*}[\mathrm{HC}-3/\mathrm{ChAT}]{$\times 1000$} & Cerebral cortex & $19.3 \pm 1.4$ & $18.4 \pm 1.1$ & $14.6 \pm 1.1$ & $14.5 \pm 1.6$ & $18.0 \pm 1.7$ & $14.5 \pm 0.6$ & $15.2 \pm 0.7$ & $13.7 \pm 0.7$ & & & \\
\hline & Midbrain & $15.2 \pm 1.1$ & $14.8 \pm 0.3$ & $13.1 \pm 0.9$ & $11.5 \pm 0.6$ & $12.5 \pm 0.7$ & $10.5 \pm 0.4 *$ & $12.3 \pm 0.7$ & $10.7 \pm 0.8$ & & & \\
\hline
\end{tabular}

Data represent means and standard errors. Asterisks denote control values for which females differ significantly from males. 
combined treatments showed a significant weight reduction relative to the group receiving prenatal nicotine alone; furthermore, the weight loss attributable to adolescent nicotine in the group exposed prenatally was indistinguishable from the magnitude of the reduction obtained in those receiving adolescent nicotine alone, so that there was no prenatal $\times$ adolescent nicotine interaction in the two-factor treatment ANOVA. There were no significant differences in brain region weights (data not shown).

Regarding treatment as a single dimension with four different levels (control, prenatal treatment, adolescent treatment, combined treatment), global ANOVA across all measures, treatments, ages, regions, and sexes identified

\section{Body Weights}

ANOVA: Rx, $p<0.0001 ; R x \times$ Age, $p<0.003 ; R x \times$ Sex, $p<0.09$

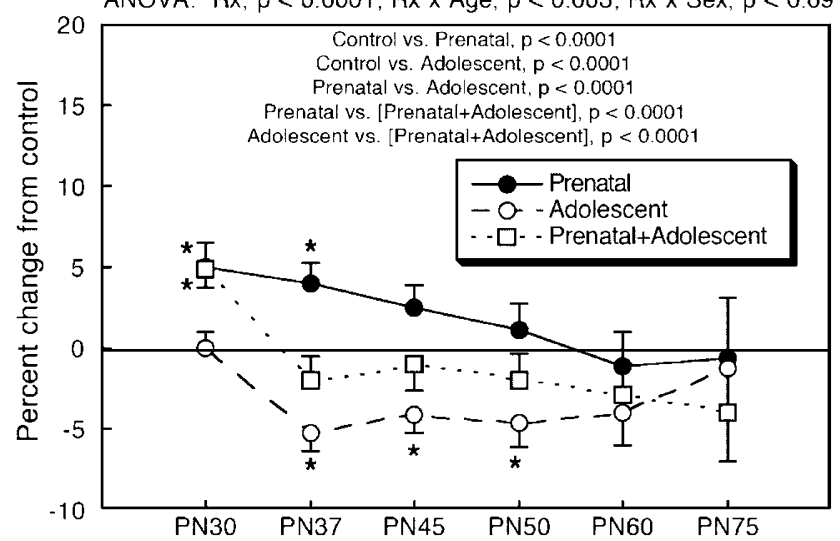

Figure I Effects of prenatal and adolescent nicotine administration on body weights, presented as the percent change from control values (see Table I). Nicotine was administered during gestation (GD4-2I), adolescence (PN30-47) or during both developmental periods. ANOVA across all treatments and ages appears at the top of the panel and intergroup comparisons are shown within the panel; asterisks denote values for which the treated groups differ significantly from the corresponding control value. Values are shown combined across males and females; although there was a treatment $\times$ sex interaction, all main effects were present in both sexes. $R x=$ treatment. significant interactions of treatment $\times$ measure and treatment $\times$ measure by other variables (Table 2 ). Similarly, regarding treatment as two dimensions (prenatal treatment, adolescent treatment), the global test identified interactions of treatment $\times$ measure for each regimen separately, as well as interactions of prenatal treatment $\times$ adolescent treatment $\times$ measure. Accordingly, the different measures were separated for presentation and were then analyzed statistically for main effects of treatment and interactions of treatment with the remaining variables.

\section{$\left[{ }^{3} \mathrm{H}\right]$ Cytisine Binding}

ANOVA across both regions detected a main treatment effect $(p<0.0001)$ as well as interactions of treatment $\times$ age $(p<0.0001)$, treatment $\times$ region $(p<0.0001)$, treatment $\times$ age $\times$ region $(p<0.03)$, and treatment $\times$ sex $\times$ region $(p<0.03)$. In the cerebral cortex (Figure 2a), prenatal nicotine exposure by itself had little or no effect on nAChRs measured in adolescence or adulthood. In contrast, adolescent nicotine administration produced robust nAChR upregulation that remained significant nearly 2 weeks after the end of treatment but subsided by PN75. Animals receiving the combined prenatal and adolescent treatment showed nAChR upregulation similar to that obtained with adolescent nicotine treatment alone; two-factor analysis of the treatment variables indicated no significant interaction of prenatal $\times$ adolescent nicotine, connoting the fact that prenatal nicotine exposure did not alter the nAChR response to adolescent nicotine administration. Although the cerebral cortex showed a significant interaction of treatment $\times$ sex, both males and females showed significant main effects of nicotine treatment $(p<0.0001)$ and interactions of treatment $\times$ age (male, $p<0.0009$; female, $p<0.0001)$. The treatment $\times$ sex interaction was confined to one age (PN60, treatment $\times$ sex, $p<0.02$ ), reflecting a slightly larger increase in the adolescent nicotine group in females $(13 \%)$ as compared to males (7\%).

In the midbrain (Figure 2b), prenatal nicotine exposure exhibited a variable effect on nAChR binding

Table 2 Global Statistical Analyses

$\mathbf{R x}$ as two dimensions: prenatal $\mathbf{R x}$, adolescent $\mathbf{R x}$

Rx as one dimension with four levels

$p<0.000$

$p<0.02$

$\mathrm{R} \times \times$ age

$\mathrm{R} \times \times$ measure

$p<0.0001$

$\mathrm{R} \times \times$ age $\times$ region

$\mathrm{R} \times \times$ age $\times$ measure

$R \times \times$ region $\times$ measure

$p<0.004$

$p<0.0001$

$R \times \times$ sex $\times$ measure

$\mathrm{R} \times \times$ age $\times$ region $\times$ measure

$\mathrm{R} \times \times$ sex $\times$ region $\times$ measure

$\mathrm{R} \times \times$ age $\times$ se $\times$ measure

\section{Prenatal Rx}

$p<0.0002$

$p<0.000$ I

$p<0.1$

$p<0.000$ ।

$p<0.09$
$p<0.06$

$p<0.09$
$p<0.06$

$p<0.03$

$\mathrm{Rx}=$ treatment. 
(treatment $\times$ age, $p<0.08$ ), with a small decrease on PN50 and an equally small increase on PN60. On the other hand, adolescent nicotine treatment again produced robust and persistent nAChR upregulation. In this region, however, prenatal nicotine exposure affected the response to adolescent nicotine treatment $(p<0.07$ for the interaction of prenatal $\times$ adolescent nicotine in a two-factor treatment design; $p<0.0001$ for the difference between adolescent nicotine and the combined treatment). The initial upregulation during adolescent nicotine administration was reduced in the animals exposed to nicotine prenatally $(p<0.02$ comparing adolescent nicotine to combined treatment on PN45) and the pretreated animals showed earlier extinction

a

nAChR Binding in Cerebral Cortex

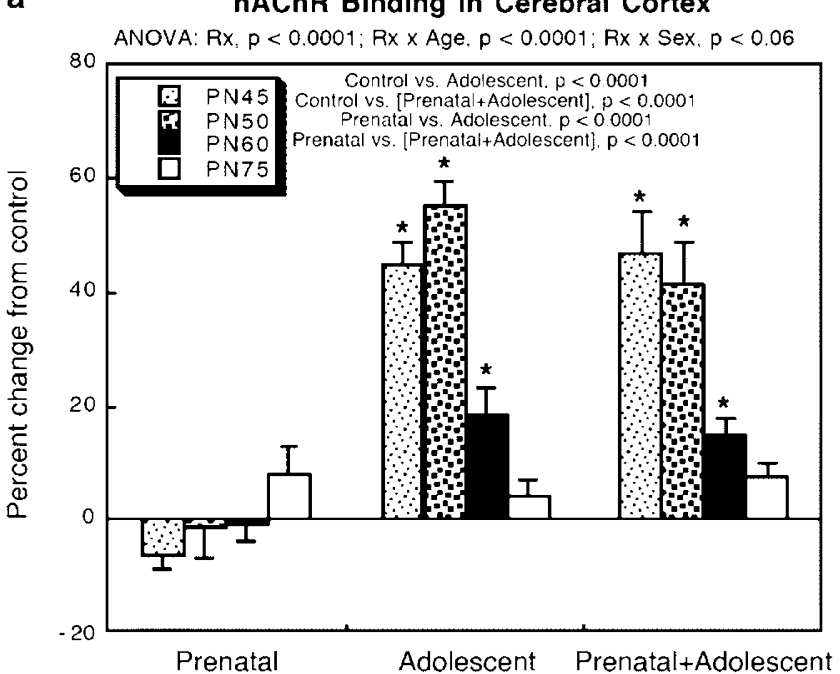

b nACh $R$ Binding in Midbrain

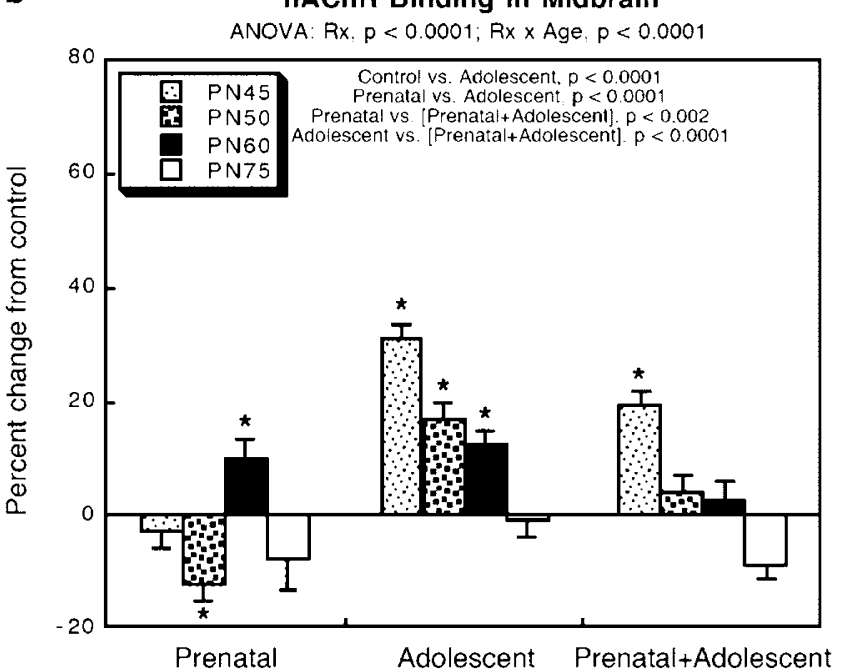

Figure 2 Effects of adolescent nicotine administration on $n A C h R$ binding in cerebral cortex (a) and midbrain (b), presented as the percent change from control values (see Table I). Nicotine was administered during gestation (GD4-2I), adolescence (PN30-47), or during both developmental periods. ANOVA across all treatments and ages appears at the top of each panel and intergroup comparisons are shown within the panels; asterisks denote values for which the treated groups differ significantly from the corresponding control value. Values are shown combined across males and females; although there was a treatment $x$ sex interaction, all main effects were present in both sexes. $R x=$ treatment. of upregulation $(p<0.0001)$. It should be noted that, although we did not separate the midbrain nAChR values by sex (since there was no treatment $\times$ sex interaction across all the ages), upregulation was more persistent in males than in females, in keeping with an earlier report (Trauth et al, 1999); on PN75, nAChRs were increased in males by about $10 \%$ whereas they were decreased in females by the same percentage $(p<0.06$ for the interaction of adolescent nicotine $\times$ sex). The lack of significant effect across sexes on PN75 thus represents the summation of the opposite effects in the two sexes.

\section{ChAT Activity (Figure 3)}

Multivariate ANOVA indicated a significant main treatment effect $(p<0.02)$ but no interactions of treatment with other variables. Accordingly, lower-order tests of separate regions, ages, and sexes were not carried out. Nicotine administration elicited global increases in ChAT activity in the prenatally exposed group $(p<0.0006)$, whereas adolescent nicotine administration had smaller effects that did not achieve statistical significance $(p<0.09)$; the greater effect of prenatal nicotine on ChAT was statistically distinguishable

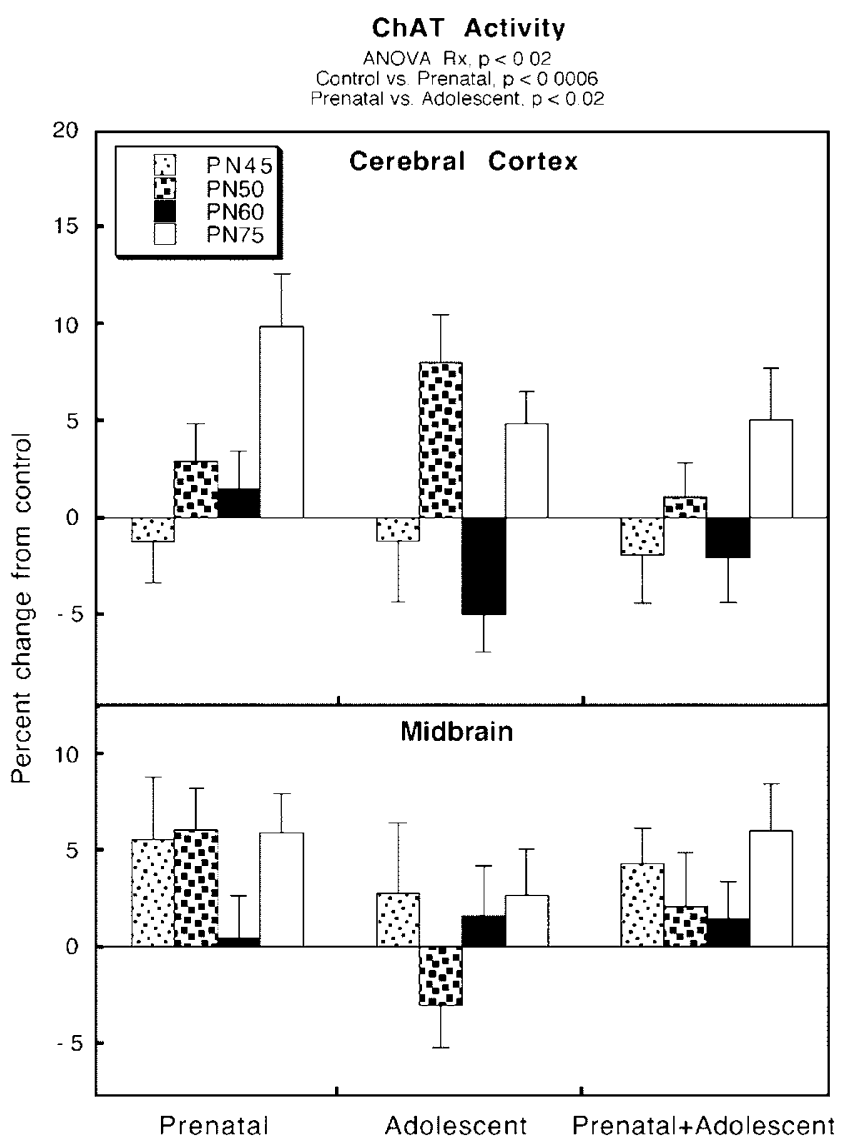

Figure 3 Effects of adolescent nicotine administration on ChAT activity, presented as the percent change from control values (see Table I). Nicotine was administered during gestation (GD4-2I), adolescence (PN30-47), or during both developmental periods. ANOVA across all treatments and ages appears at the top of the panel; lower-order tests were not carried out because of the absence of treatment interactions with region, sex, or age. Values are shown combined across males and females. $\mathrm{R} \times=$ treatment. 
from the smaller effect of adolescent nicotine $(p<0.02)$. The group receiving combined treatment likewise showed a smaller elevation that did not reach the significance criterion $(p<0.08)$, but in this case the effect was less than would have been expected from additive actions of the two nicotine treatments $(p<0.08$ for the interaction of prenatal $\times$ adolescent treatment in the two-factor treatment ANOVA). Importantly, increases in ChAT activity elicited either by prenatal nicotine alone or by the combined prenatal + adolescent treatment remained detectable on PN75 $(p<0.0006$ and $<0.02$, respectively) more than 2 months after the end of prenatal exposure and 1 month after the termination of adolescent treatment.

\section{HC-3 Binding}

In the absence of any changes in neural activity, HC-3 binding should mimic the changes seen for ChAT. Global ANOVA detected a main treatment effect $(p<0.0001)$ and additional interactions of treatment $\times$ region $(p<0.03)$ and treatment $\times$ age $\times$ region $(p<0.02)$, but the direction of the main effect was for overall decreases in HC-3 binding, opposite to the direction seen for ChAT. In the light of the interactions, the data were subdivided into separate regions for lower-order analysis. In the cerebral cortex, there was an overall treatment effect as well as an interaction of treatment $\times$ age (Figure $4 \mathrm{a}$ ). All three treatment paradigms elicited significant overall reductions in HC-3 binding compared to control values (main effect). The two-factor treatment analysis indicated a significant interaction of prenatal exposure $\times$ adolescent treatment $\times$ age, indicative of effects that could not be accounted for by simple additivity of the two individual treatments, and reflecting primarily the less-than-additive effects on PN45 $(p<0.004)$. However, for the subsequent ages during nicotine withdrawal, the effects were not distinguishable from additivity of prenatal and adolescent nicotine.

In the midbrain, global ANOVA again indicated significant overall reductions that were individually significant for each of the three treatment regimens (Figure 4b). The effect of prenatal nicotine exposure was marginally greater than that of adolescent treatment $(p<0.07)$ and the effect of combined treatment was significantly greater than that of adolescent treatment alone; however, the net effect was no greater than the additive effects of the two separate treatments (no interaction of prenatal $\times$ adolescent nicotine in the two-factor treatment analysis).

As HC-3 binding is dependent both upon the density of cholinergic innervation and on neural activity, we also assessed the binding as the $\mathrm{HC}-3 / \mathrm{ChAT}$ ratio. With this correction, effects in the cerebral cortex were sex-dependent (interaction of treatment $\times$ sex), necessitating separation of values for males and females, although significant differences were seen for both sexes (Figure 5a). In males, prenatal nicotine exposure produced overall reductions in the binding ratio, as did adolescent nicotine treatment or the combination of the two treatments. The effects were less-than-additive during nicotine administration (PN45, $p<0.0007$ for the interaction of prenatal exposure $\times$ adolescent treatment). During withdrawal, however, the effects of combined exposure were greater than those of prenatal or adolescent nicotine alone, and the net effect was
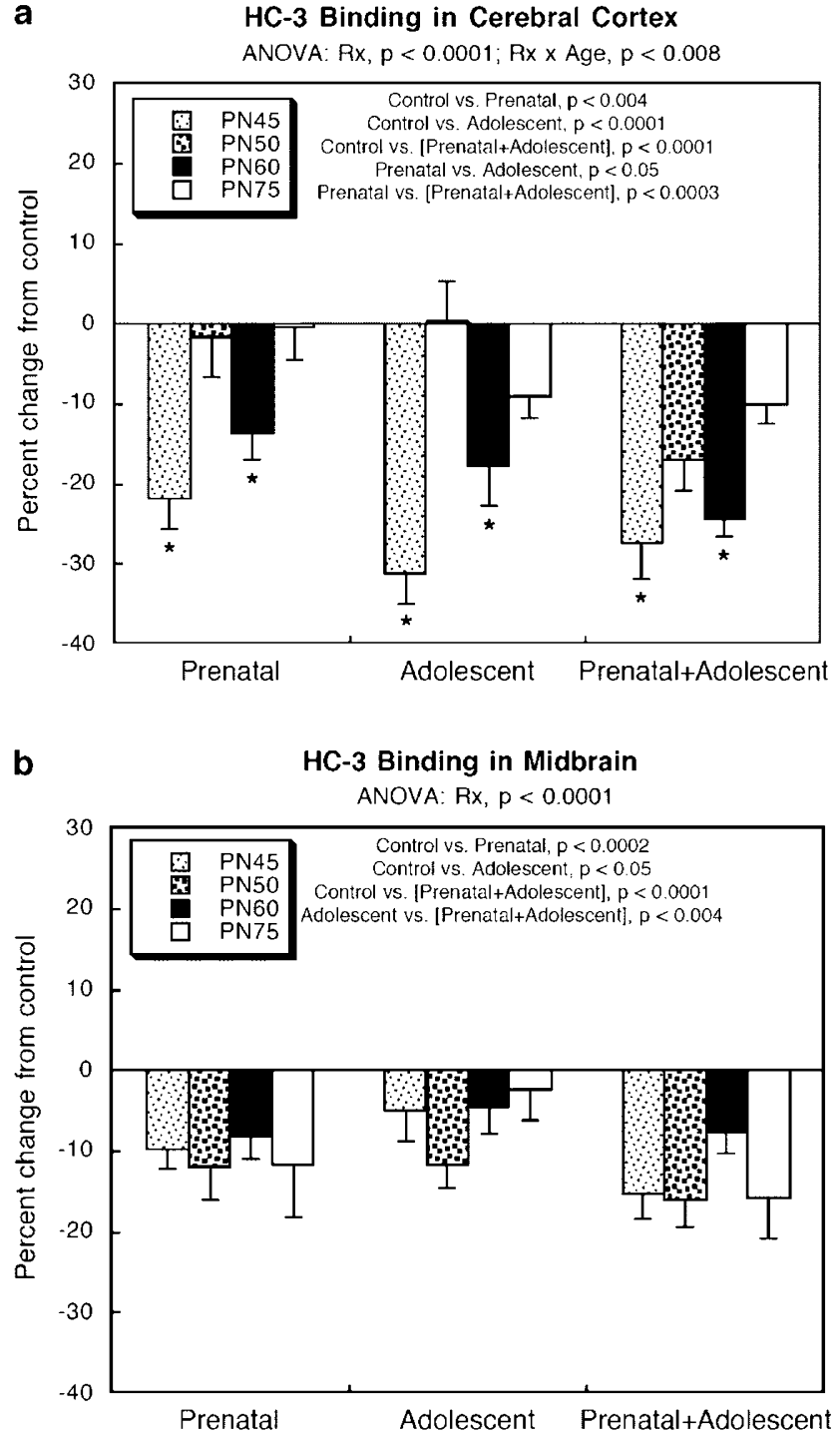

Figure 4 Effects of adolescent nicotine administration on $\left.{ }^{3} \mathrm{H}\right] \mathrm{HC}-3$ binding in cerebral cortex (a) and midbrain (b), presented as the percent change from control values (see Table I). Nicotine was administered during gestation (GD4-2I), adolescence (PN30-47), or during both developmental periods. ANOVA across all treatments and ages appears at the top of each panel and intergroup comparisons are shown within the panels. For the cerebral cortex, asterisks denote ages for which the treated groups differ significantly from the corresponding control value; for the midbrain, only main treatment effects are shown because of the absence of a treatment $\times$ age interaction. Values are shown combined across males and females because of the absence of interactions of treatment $x$ sex or treatment $\times \operatorname{sex} \times$ age. $R \times=$ treatment.

not distinguishable from additivity. In females, the effects of prenatal nicotine exposure alone were smaller and nonsignificant overall when compared to control values; the difference between male and female effects was significant (prenatal exposure $\times$ sex, $p<0.05$ ). Adolescent nicotine suppressed the HC-3/ChAT ratio overall in females, although again, the effect was significantly lower than that seen in males (adolescent treatment $\times$ sex, $p<0.03$ ). The combination of prenatal exposure and adolescent treatment likewise evoked a decrement in the activity ratio in females but in this case, the effects were indistinguishable in males 
a

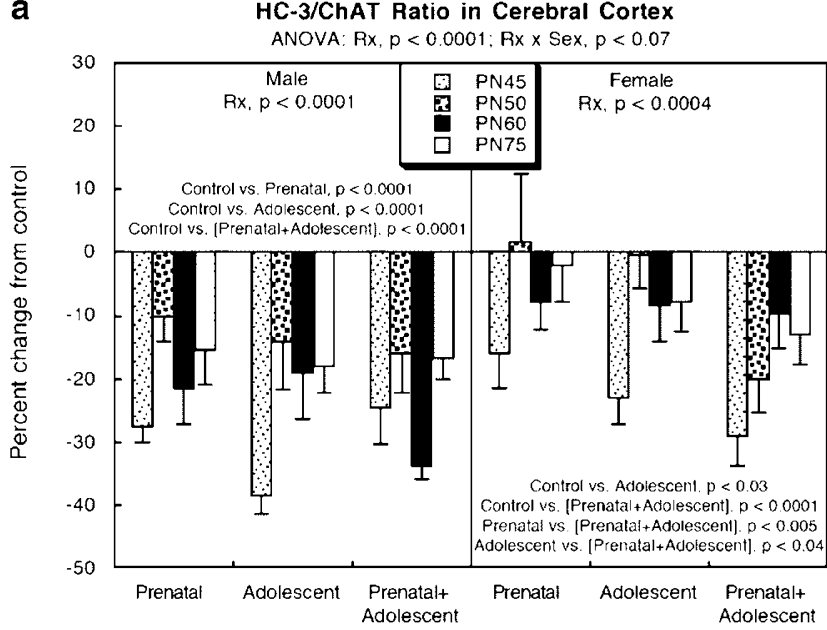

b

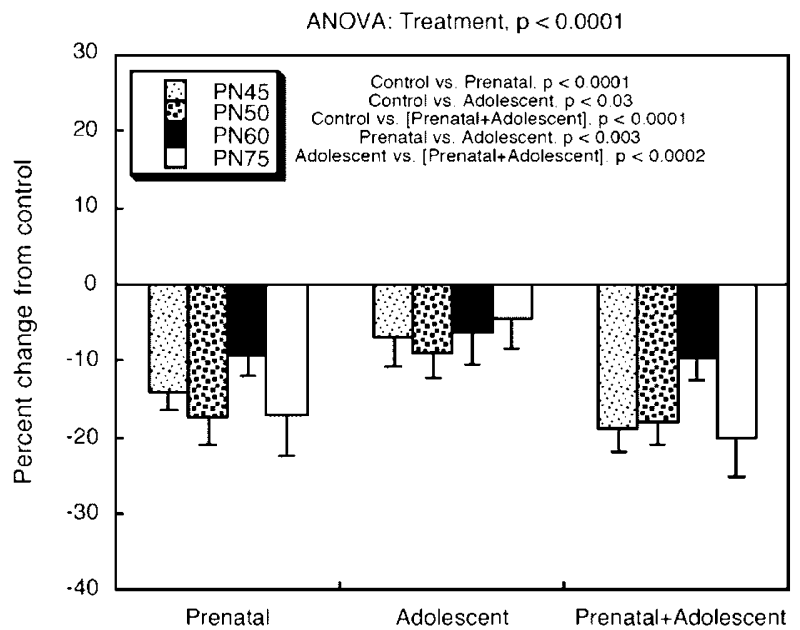

Figure 5 Effects of adolescent nicotine administration on the $\mathrm{HC}-3 /$ ChAT ratio in cerebral cortex (a) and midbrain (b), presented as the percent change from control values (see Table I). Nicotine was administered during gestation (GD4-2I), adolescence (PN30-47), or during both developmental periods. ANOVA across all treatments and ages appears at the top of each panel and intergroup comparisons are shown within the panels. For the cerebral cortex, ANOVA indicated sexdependent effects (treatment $x$ sex interaction, necessitating separation of values for males and females, whereas for the midbrain, the values for both sexes were combined. Only main treatment effects are shown because of the absence of treatment $\times$ age interactions. $R \times=$ treatment.

and females (no treatment $\times$ sex interaction). For the combined treatment regimen in females, two-factor analysis of treatment effects indicated additivity of the prenatal and adolescent effects (no interaction of prenatal $\times$ adolescent nicotine).

In contrast to the sex-dependent effects on the cerebrocortical HC-3/ChAT ratio, effects in the midbrain did not show a significant treatment $\times$ sex interaction but did show the same overall lowering of activity (Figure $5 b$ ). Again, both prenatal and adolescent nicotine by themselves elicited significant overall reductions that persisted for months after the termination of nicotine exposure, with a greater effect of the prenatal regimen. The combination of prenatal exposure and adolescent treatment also produced significant, persistent decrements that were not distinguishable from the summation of the two individual effects (no prenatal $\times$ adolescent treatment interaction).

\section{DISCUSSION}

Results obtained in this study are consistent with the view that prenatal nicotine exposure disrupts the programming of cholinergic function in adolescence and adulthood (Levin and Slotkin, 1998; Slotkin, 1992, 1998, 1999, 2004), and that the vulnerability of cholinergic systems to persistent disruption by nicotine continues into adolescence (AbreuVillaça et al, 2003a; Slotkin, 2002; Trauth et al, 1999, 2000a). Furthermore, the current results indicate that prenatal nicotine exposure alters the response to nicotine administered during adolescence. In the light of epidemiological findings relating maternal smoking during pregnancy with adolescent smoking in the offspring (Cornelius et al, 2000; Kandel et al, 1994; Niaura et al, 2001), our findings provide evidence for biological mechanisms underlying this association, reflecting alterations evoked by prenatal nicotine exposure. In the ensuing paragraphs we elaborate the essential conclusions and propose a mechanism by which prenatal nicotine exposure may predispose adolescents to nicotine addiction.

Earlier work showed that prenatal nicotine exposure elicits short-term upregulation of nAChRs in brain regions of the offspring, with a return to control values in the first few weeks postpartum (Slotkin et al, 1987). By adulthood, neither the mRNA encoding nAChR subunits nor their corresponding proteins are altered in the broadly defined brain regions used here (Shacka and Robinson, 1998; Van de Kamp and Collins, 1994). In the course of the transition from adolescence to adulthood, we did observe small, but significant, fluctuations in nAChRs in the midbrain, likely reflecting more focal changes in specific subnuclei (Tizabi and Perry, 2000) but by full adulthood (PN75) nAChR parameters were essentially normal in the prenatal nicotine group. Nevertheless, the prenatal exposure altered the ability of adolescent nicotine to evoke nAChR upregulation in the midbrain, evidenced by a reduced effect during the period of nicotine administration and a more rapid return to normal after discontinuing the adolescent treatment; indeed, the animals exposed to prenatal nicotine and then given nicotine in adolescence showed no nAChR upregulation by PN50, only 2 days after discontinuing nicotine administration, whereas values remained elevated for at least 2 weeks post-treatment in the group receiving adolescent nicotine alone (and actually for 1 month in males, as noted above).

The obtunded response in adolescence after prenatal nicotine exposure did not reflect pharmacokinetic differences elicited by the fetal treatment; accelerated catabolism of nicotine (and hence lower effective levels for the same dose) would also have reduced the somatic growth inhibition evoked by adolescent nicotine, but this was not seen. Instead, then, our results suggest a specific impairment of nAChR upregulation in response to adolescent treatment in the group exposed to nicotine prenatally. Upregulation of $\alpha 4 \beta 2 \mathrm{nAChRs}$ is initiated by receptor desensitization (Fenster et al, 1999) and both desensitization and upregulation contribute to nicotine addiction 
(Buisson and Bertrand, 2002; Dani and De Biasi, 2001; Quick and Lester, 2002). Our results therefore suggest two mutually exclusive scenarios. Prenatal exposure might reduce the adolescent response to nicotine, thus leading to a lesser degree of desensitization and nAChR upregulation. Alternatively, if adolescent nicotine elicits its full effect, then reduced degrees of desensitization and receptor upregulation could connote a greater net biological response. The former explanation is almost certainly true, since direct assessment of CNS catecholamine release evoked by acute nicotine challenge is reduced in adolescent rats exposed to nicotine prenatally (Seidler et al, 1992). Given the critical role of midbrain circuitry in mechanisms of addiction (Mansvelder and McGehee, 2002; Nestler, 2001), the desensitization evoked by fetal nicotine exposure suggests a lasting biological difference in this aspect of adolescent nicotine effects.

We also evaluated whether prenatal nicotine exposure altered the ability of adolescent nicotine to influence cholinergic synaptic function. For ChAT activity, prenatal nicotine elicited a small but consistent increase throughout adolescence and adulthood. Nicotine itself does not directly alter ChAT expression or enzymatic activity, nor does nicotine treatment in adulthood affect ChAT (Lapchak et al, 1989; Wessler et al, 2003). Furthermore, as a constitutive biomarker for cholinergic synapses, ChAT largely reflects the concentration of cholinergic terminals and is insensitive to changes in neural impulse activity (Aubert et al, 1996; Happe and Murrin, 1992; Navarro et al, 1989; Slotkin et al, 1990; Zahalka et al, 1992, 1993). Accordingly, our results suggest that prenatal nicotine exposure elicits a small but significant increase in the density of cholinergic innervation, likely in reaction to adverse effects on neuronal size and the relative thickness of the neuropil that are detectable in the period immediately preceding and during the increase in ChAT (Roy and Sabherwal, 1994, 1998; Roy et al, 2002). Indeed, the effect on ChAT emerges rather late in development, as it is not present in the peripubertal period or early adolescence (Navarro et al, 1989); as seen here, the increase in the cerebral cortex showed an onset only with the approach of adulthood. The effect was also demonstrable with adolescent nicotine administration, albeit smaller in magnitude. We were unable to demonstrate an increased effect from the combination of the two nicotine treatments, but given the small magnitude of the effects, this may represent a limit of statistical power rather than the absence of a biological interaction. In fact, as discussed next, the major effects were seen for biomarkers of synaptic activity rather than synaptic integrity.

If effects on the concentration of synaptic terminals were the only effect of prenatal nicotine on cholinergic systems, then all biomarkers associated with the terminals would show the same pattern. This was clearly not the case, as HC3 binding was significantly reduced, both in absolute terms and when evaluated as the $\mathrm{HC}-3 / \mathrm{ChAT}$ ratio. $\mathrm{HC}-3$, unlike ChAT, is highly regulated by neural impulse activity (Aubert et al, 1996; Bissette et al, 1996; Happe and Murrin, 1992; Klemm and Kuhar, 1979; Navarro et al, 1989; Simon et al, 1976; Slotkin et al, 1990; Zahalka et al, 1992, 1993), so the disparity in the two effects suggests an impairment of cholinergic tone in the animals exposed to prenatal nicotine. Again, these effects emerge only after a period of relative normality, since they are not present at younger stages (Navarro et al, 1989), suggesting developmental disruption of the programming of cholinergic activity rather than a continuous effect ongoing from the prenatal exposure period. Previous studies of HC-3 binding in the hippocampus likewise show delayed-onset deficits (Zahalka et al, 1992). Adolescent nicotine exposure by itself also elicited persistent reductions in this index of neural activity, with about the same magnitude of effect as seen with prenatal nicotine, in keeping with earlier results (AbreuVillaça et al, 2003a; Trauth et al, 2000a). When animals exposed to nicotine prenatally were then given nicotine in adolescence, unlike the situation for nAChRs, the effects on HC-3 binding showed equivalent or additive effects during the withdrawal period. In only one case, the cerebral cortex in males, the effects on the $\mathrm{HC}-3 / \mathrm{ChAT}$ ratio were less-thanadditive but that may be a reflection of the larger overall magnitude of the deficits in that group; it may not be possible to reduce activity further much beyond $30 \%$, given that total arrest of neural activity by deafferentation or general anesthesia reduces choline transporter function only slightly more (Murrin et al, 1977; Simon et al, 1976). Additivity of the effects of prenatal and adolescent nicotine were clearly demonstrated in the cerebral cortex in females, where the combined treatment elicited distinguishably greater deficits than either treatment alone. Similarly, in the midbrain, the combined effect was statistically larger than that of adolescent nicotine. Accordingly, although prenatal nicotine exposure diminishes the subsequent ability of the adolescent brain to upregulate nAChRs in response to nicotine, it does not interfere globally with the reductions in cholinergic synaptic activity elicited by adolescent nicotine, and instead, the effects of the two exposures can be cumulative.

In sum, the present results indicate effects of prenatal nicotine exposure on cholinergic systems that persist into adolescence, the stage at which smoking typically commences. If similar effects occur in the offspring of women who smoke during pregnancy, then a number of our findings may point to biological mechanisms that underlie the increased likelihood of nicotine addiction in adolescence (Cornelius et al, 2000; Kandel et al, 1994; Niaura et al, 2001). First, the functional consequences of chronic cholinergic hypoactivity may provide one of the essential elements that reinforce the effects of nicotine: by selfadministering the drug, the affected adolescent supplies exogenous cholinergic stimulation that replaces the functional loss. However, we also found deficiencies in $\mathrm{nAChR}$ upregulation in adolescence after prenatal nicotine exposure, effects that, along with earlier assessments (Seidler et al, 1992) indicated desensitization of CNS responses to nicotine. The deficient response to nicotinic stimulation in adolescents whose mothers smoked during pregnancy may then drive higher cigarette consumption (Chassin et al, 1996; Chen and Millar, 1998). We also observed greater deficits in cholinergic synaptic activity with the combination of prenatal and adolescent nicotine; accordingly, the impact of adolescent smoking on endogenous cholinergic activity may be worsened in the offspring of smokers. Indeed, since craving and relapse involve plasticity not only in the midbrain but also in the cerebral cortex (Nestler, 2001), the summation of cholinergic deficits evoked by the 
prenatal and adolescent exposures in the cerebral cortex of females may be one of the reasons why maternal smoking during pregnancy is a stronger predictor of smoking in adolescent women (Cornelius et al, 2000; Kandel et al, 1994; Niaura et al, 2001). Finally, the effects of both prenatal and adolescent nicotine on biomarkers of cholinergic synaptic activity were extremely persistent, lasting into adulthood, more than 2 months after the termination of prenatal nicotine exposure and a month after the end of adolescent nicotine treatment. Although the present results do not identify a specific mechanism underlying these persistent alterations, there are two likely possibilities based on the known effects of nicotine on the developing brain (Levin and Slotkin, 1998; Slotkin, 1992, 1998, 1999, 2002, 2004; Yanai et al, 2002). First, nicotine alters the programming of synaptic responsiveness, eliciting long-term changes in patterns of neural activity and postsynaptic cell signaling. Second, nicotine is a developmental neurotoxicant that evokes neuronal and synaptic damage, effects that would certainly contribute to persistent or permanent alterations in synaptic performance. Indeed, neurotoxicant injury from adolescent nicotine administration is greater in females than in males, mirroring some of the synaptic changes seen here (Slotkin, 2002; Trauth et al, 2000b; Xu et al, 2003). Regardless of the mechanism, however, the lasting alterations seen in the current study may contribute to functional deficits that persist beyond the initial stages of withdrawal in adolescent smokers (DiFranza et al, 2000, 2002a,b; O'Loughlin et al, 2002), producing cognitive or other perturbations that ultimately contribute to relapse. Our findings in rats thus reinforce the observations made in adolescent smokers and in the subpopulation of adolescents whose mothers smoked during pregnancy and point to a specific biological basis for the increased susceptibility to nicotine addiction.

\section{ACKNOWLEDGEMENTS}

Supported by USPHS DA14247, by a grant and fellowship from Philip Morris USA Inc., and by a fellowship from $\mathrm{CNPq}$ Brazil. We thank Indira Thillai for technical assistance.

\section{REFERENCES}

Abreu-Villaça Y, Seidler FJ, Qiao D, Tate CA, Cousins MM, Thillai I et al (2003a). Short-term adolescent nicotine exposure has immediate and persistent effects on cholinergic systems: critical periods, patterns of exposure, dose thresholds. Neuropsychopharmacology 28: 1935-1949.

Abreu-Villaça Y, Seidler FJ, Slotkin TA (2003b). Impact of adolescent nicotine exposure on adenylyl cyclase-mediated cell signaling: enzyme induction, neurotransmitter-specific effects, regional selectivities, and the role of withdrawal. Brain Res 988: 164-172.

Abreu-Villaça Y, Seidler FJ, Tate CA, Slotkin TA (2003c). Nicotine is a neurotoxin in the adolescent brain: critical periods, patterns of exposure, regional selectivity, and dose thresholds for macromolecular alterations. Brain Res 979: 114-128.

Adriani W, Macri S, Pacifici R, Laviola G (2002). Peculiar vulnerability to nicotine oral self-administration in mice during early adolescence. Neuropsychopharmacology 27: 212-224.
Adriani W, Spijker S, Deroche-Gamonet V, Laviola G, Le Moal M, Smit $A B$ et al (2003). Evidence for enhanced neurobehavioral vulnerability to nicotine during periadolescence in rats. J Neurosci 23: 4712-4716.

Aubert I, Cecyre D, Gauthier S, Quirion R (1996). Comparative ontogenic profile of cholinergic markers, including nicotinic and muscarinic receptors, in the rat brain. J Comp Neurol 369: 31-55.

Bauman KE, Foshee VA, Linzer MA, Koch GG (1990). Effect of parental smoking classification on the association between parental and adolescent smoking. Addict Behav 15: 413-422.

Bissette G, Seidler FJ, Nemeroff CB, Slotkin TA (1996). High affinity choline transporter status in Alzheimer's Disease tissue from rapid autopsy. Ann NY Acad Sci 777: 197-204.

Buisson B, Bertrand D (2002). Nicotine addiction: the possible role of functional upregulation. Trends Pharmacol Sci 23: 130-136.

Centers for Disease Control and Prevention (1998). Tobacco use among high school students-United States, 1997. Morbid Mortal Weekly Rep 47: 229-233.

Chassin L, Presson CC, Rose JS, Sherman SJ (1996). The natural history of cigarette smoking from adolescence to adulthood: demographic predictors of continuity and change. Health Psychol 15: 478-484.

Chassin L, Presson CC, Rose JS, Sherman SJ, Prost J (2002). Parental smoking cessation and adolescent smoking. If Pediatr Psychol 27: 495-496.

Chen J, Millar WJ (1998). Age of smoking inititation: implications for quitting. Health Rep 9: 39-46.

Cornelius MD, Leech SL, Goldschmidt L, Day NL (2000). Prenatal tobacco exposure: is it a risk factor for early tobacco experimentation? Nicotine Tobacco Res 2: 45-52.

Dani JA, De Biasi M (2001). Cellular mechanisms of nicotine addiction. Pharmacol Biochem Behav 70: 439-446.

DiFranza J, Savageau JA, Rigotti NA, Fletcher K, Ockene JK, McNeill AD et al (2002a). Development of symptoms of tobacco dependence in youths: 30-month follow-up data from the DANDY study. Tobacco Control 11: 228-235.

DiFranza JR, Rigotti NA, McNeill AD, Ockene JK, Savageau JA, St. Cyr D et al (2000). Initial symptoms of nicotine dependence in adolescents. Tobacco Control 9: 313-319.

DiFranza JR, Savageau JA, Fletcher K, Ockene JK, Rigotti NA, $\mathrm{McNeill} \mathrm{AD}$ et al (2002b). Measuring the loss of autonomy over nicotine use in adolescents: the DANDY (development and assessment of nicotine dependence in youths) study. Arch Pediatr Adolesc Med 156: 397-403.

Faraday MM, Elliott BM, Grunberg NE (2001). Adult $v s$ adolescent rats differ in biobehavioral responses to chronic nicotine administration. Pharmacol Biochem Behav 70: 475-489.

Faraday MM, Elliott BM, Phillips JM, Grunberg NE (2003). Adolescent and adult male rats differ in sensitivity to nicotine's activity effects. Pharmacol Biochem Behav 74: 917-931.

Fenster CP, Whitworth TL, Sheffield EB, Quick MW, Lester RAJ (1999). Upregulation of surface $\alpha_{4} \beta_{2}$ nicotinic receptors is initiated by receptor desensitization after chronic exposure to nicotine. J Neurosci 19: 4804-4814.

Flores CM, Rogers SW, Pabreza LA, Wolfe BB, Kellar KJ (1992). A subtype of nicotinic cholinergic receptor in rat brain is composed of $\alpha 4$ and $\beta 2$ subunits and is upregulated by chronic nicotine treatment. Mol Pharmacol 41: 31-37.

Happe HK, Murrin LC (1992). High-affinity choline transport regulation by drug administration during postnatal development. J Neurochem 58: 2053-2059.

Happe HK, Peters JL, Bergman DA, Murrin LC (1994). Localization of nicotinic cholinergic receptors in rat brain: autoradiographic studies with $\left[{ }^{3} \mathrm{H}\right]$ cytisine. Neuroscience 62: 929-944.

Kandel DB, Wu P, Davies M (1994). Maternal smoking during pregnancy and smoking by adolescent daughters. Am J Public Health 84: 1407-1413. 
Klein LC, Stine MM, Pfaff DW, Vandenbergh DJ (2003). Maternal nicotine exposure increases nicotine preference in periadolescent male but not female C57B1/6J mice. Nicotine Tobacco Res 5: 117-124.

Klemm N, Kuhar MJ (1979). Post-mortem changes in high affinity choline uptake. J Neurochem 32: 1487-1494.

Lapchak PA, Araujo DM, Quirion R, Collier B (1989). Effect of chronic nicotine treatment on nicotinic autoreceptor function and $\mathrm{N}-\left[{ }^{3} \mathrm{H}\right]$ methylcarbamylcholine binding sites in the rat brain. J Neurochem 52: 483-491.

Lau C, Seidler FJ, Cameron AM, Navarro HA, Bell JM, Bartolome J et al (1988). Nutritional influences on adrenal chromaffin cell development: comparison with central neurons. Pediatr Res 24: 583-587.

Levin ED (1999). Persisting effects of chronic adolescent nicotine administration on radial-arm maze learning and response to nicotinic challenges. Neurotoxicol Teratol 21: 338.

Levin ED, Slotkin TA (1998). Developmental neurotoxicity of nicotine. In: Slikker W, Chang LW (eds). Handbook of Developmental Neurotoxicology. Academic Press: San Diego. pp 587-615.

Lichtensteiger W, Ribary U, Schlumpf M, Odermatt B, Widmer HR (1988). Prenatal adverse effects of nicotine on the developing brain. Prog Brain Res 73: 137-157.

Mansvelder HD, McGehee DS (2002). Cellular and synaptic mechanisms of nicotine addiction. J Neurobiol 53: 606-617.

Murrin LC, DeHaven RN, Kuhar MJ (1977). On the relationship between $(3 \mathrm{H})$ choline uptake activation and $(3 \mathrm{H})$ acetylcholine release. J Neurochem 29: 681-687.

Murrin LC, Ferrer JR, Wanyun Z, Haley NJ (1987). Nicotine administration to rats: methodological considerations. Life Sci 40: $1699-1708$.

National Institute on Drug Abuse (1998). Nicotine Addiction, NIH Publication Number 98-4342: Washington, DC, 7pp.

Navarro HA, Seidler FJ, Eylers JP, Baker FE, Dobbins SS, Lappi SE et al (1989). Effects of prenatal nicotine exposure on development of central and peripheral cholinergic neurotransmitter systems. Evidence for cholinergic trophic influences in developing brain. J Pharmacol Exp Ther 251: 894-900.

Nelson DE, Giovino GA, Shopland DR, Mowery PD, Mills SL, Eriksen MP (1995). Trends in cigarette smoking among US adolescents, 1974 through 1991. Am J Public Health 85: $34-40$.

Nestler EJ (2001). Molecular basis of long-term plasticity underlying addiction. Nature Rev Neurosci 2: 119-128.

Niaura R, Bock B, Lloyd EE, Brown R, Lipsitt LP, Buka S (2001). Maternal transmission of nicotine dependence: psychiatric, neurocognitive and prenatal factors. Am J Addict 10: $16-29$.

O'Loughlin J, Kishchuk N, DiFranza J, Tremblay M, Paradis G (2002). The hardest thing is the habit: a qualitative investigation of adolescent smokers' experience of nicotine dependence. Nicotine Tobacco Res 4: 201-209.

Ostrea EM, Knapp DK, Romero A, Montes M, Ostrea AR (1994). Meconium analysis to assess fetal exposure to nicotine by active and passive maternal smoking. J Pediatr 124: 471-476.

Pierce JP, Gilpin E (1996). How long will today's new adolescent smoker be addicted to cigarettes? Am J Public Health 86: 253-256.

Quick MW, Lester RA (2002). Desensitization of neuronal nicotinic receptors. J Neurobiol 53: 457-478.

Ribary U, Lichtensteiger W (1989). Effects of acute and chronic prenatal nicotine treatment on central catecholamine systems of male and female rat fetuses and offspring. J Pharmacol Exp Ther 248: 786-792.

Roy TS, Sabherwal U (1994). Effects of prenatal nicotine exposure on the morphogenesis of somatosensory cortex. Neurotoxicol Teratol 16: 411-421.
Roy TS, Sabherwal U (1998). Effects of gestational nicotine exposure on hippocampal morphology. Neurotoxicol Teratol 20: $465-473$.

Roy TS, Seidler FJ, Slotkin TA (2002). Prenatal nicotine exposure evokes alterations of cell structure in hippocampus and somatosensory cortex. J Pharmacol Exp Ther 300: 124-133.

Seidler FJ, Levin ED, Lappi SE, Slotkin TA (1992). Fetal nicotine exposure ablates the ability of postnatal nicotine challenge to release norepinephrine from rat brain regions. Dev Brain Res 69: 288-291.

Shacka JJ, Robinson SE (1998). Exposure to prenatal nicotine transiently increases neuronal nicotinic receptor subunit alpha7, alpha-4 and beta-2 messenger RNAs in the postnatal rat brain. Neuroscience 84: 1151-1161.

Simon JR, Atweh S, Kuhar MJ (1976). Sodium-dependent high affinity choline uptake: a regulatory step in the synthesis of acetylcholine. J Neurochem 26: 909-922.

Slawecki CJ, Ehlers CL (2002). Lasting effects of adolescent nicotine exposure on the electroencephalogram, event related potentials, and locomotor activity in the rat. Dev Brain Res 138: $15-25$.

Slotkin TA (1992). Prenatal exposure to nicotine: what can we learn from animal models?. In: Zagon IS, Slotkin TA (eds). Maternal Substance Abuse and the Developing Nervous System. Academic Press: San Diego. pp 97-124.

Slotkin TA (1998). Fetal nicotine or cocaine exposure: which one is worse? J Pharmacol Exp Ther 285: 931-945.

Slotkin TA (1999). Developmental cholinotoxicants: nicotine and chlorpyrifos. Environ Health Perspectives 107(Suppl 1): 71-80.

Slotkin TA (2002). Nicotine and the adolescent brain: insights from an animal model. Neurotoxicol Teratol 24: 369-384.

Slotkin TA (2004). Cholinergic systems in brain development and disruption by neurotoxicants: nicotine, environmental tobacco smoke, organophosphates. Toxicol Appl Pharmacol, in press.

Slotkin TA, Orband-Miller L, Queen KL (1987). Development of $\left[{ }^{3} \mathrm{H}\right]$ nicotine binding sites in brain regions of rats exposed to nicotine prenatally via maternal injections or infusions. J Pharmacol Exp Ther 242: 232-237.

Slotkin TA, Seidler FJ, Crain BJ, Bell JM, Bissette G, Nemeroff CB (1990). Regulatory changes in presynaptic cholinergic function assessed in rapid autopsy material from patients with Alzheimer disease: implications for etiology and therapy. Proc Natl Acad Sci USA 87: 2452-2455.

Smith PK, Krohn RI, Hermanson GT, Mallia AK, Gartner FH, Provenzano MD et al (1985). Measurement of protein using bicinchoninic acid. Anal Biochem 150: 76-85.

Snedecor GW, Cochran WG (1967). Statistical Methods. Iowa State University Press: Ames, IA. 593pp.

Tizabi Y, Perry DC (2000). Prenatal nicotine exposure is associated with an increase in $\left[{ }^{125} \mathrm{I}\right]$ epibatidine binding in discrete cortical regions in rats. Pharmacol Biochem Behav 67: 319-323.

Trauth JA, McCook EC, Seidler FJ, Slotkin TA (2000a). Modeling adolescent nicotine exposure: effects on cholinergic systems in rat brain regions. Brain Res 873: 18-25.

Trauth JA, Seidler FJ, Ali SF, Slotkin TA (2001). Adolescent nicotine exposure produces immediate and long-term changes in CNS noradrenergic and dopaminergic function. Brain Res 892: 269-280.

Trauth JA, Seidler FJ, McCook EC, Slotkin TA (1999). Adolescent nicotine exposure causes persistent upregulation of nicotinic cholinergic receptors in rat brain regions. Brain Res 851: 9-19.

Trauth JA, Seidler FJ, Slotkin TA (2000b). An animal model of adolescent nicotine exposure: effects on gene expression and macromolecular constituents in rat brain regions. Brain Res 867: 29-39.

Trauth JA, Seidler FJ, Slotkin TA (2000c). Persistent and delayed behavioral changes after nicotine treatment in adolescent rats. Brain Res 880: 167-172. 
Van de Kamp JL, Collins AC (1994). Prenatal nicotine alters nicotinic receptor development in the mouse brain. Pharmacol Biochem Behav 47: 889-900.

Wessler I, Schwarze S, Brockerhoff P, Bittinger F, Kirkpatrick CJ, Kilbinger H (2003). Effects of sex hormones, forskolin, and nicotine on choline acetyltransferase activity in human isolated placenta. Neurochem Res 28: 489-492.

Whiting P, Lindstrom J (1987). Purification and characterization of a nicotinic acetylcholine receptor from rat brain. Proc Natl Acad Sci 84: 595-599.

Whiting PR, Lindstrom J (1988). Characterization of bovine and human neuronal nicotinic acetylcholine receptors using monoclonal antibodies. J Neurosci 8: 3395-3404.

Xu Z, Seidler FJ, Tate CA, Garcia SJ, Slikker W, Slotkin TA (2003). Sex-selective hippocampal alterations after adolescent nicotine

administration: effects on neurospecific proteins. Nicotine Tobacco Res 5: 955-960.

Yanai J, Vatury O, Slotkin TA (2002). Cell signaling as a target and underlying mechanism for neurobehavioral teratogenesis. Ann NY Acad Sci 965: 473-478.

Zahalka E, Seidler FJ, Lappi SE, Yanai J, Slotkin TA (1993). Differential development of cholinergic nerve terminal markers in rat brain regions: implications for nerve terminal density, impulse activity and specific gene expression. Brain Res 601: 221-229.

Zahalka EA, Seidler FJ, Lappi SE, McCook EC, Yanai J, Slotkin TA (1992). Deficits in development of central cholinergic pathways caused by fetal nicotine exposure: differential effects on choline acetyltransferase activity and $\left[{ }^{3} \mathrm{H}\right]$ hemicholinium-3 binding. Neurotoxicol Teratol 14: 375-382. 